\title{
HACIA UN SISTEMA COMPLEMENTARIO DE PRODUCCIÓN MÁS LIMPIA EN SUELOS DEGRADADOS POR SALINIDAD
}

\author{
Toward a complementary system of cleaner production on \\ soils degraded by salinity
}

RAMIRO CUERO 1

1Universidad del Valle

E-mail: racuero@univalle.edu.co

Recibido: 1 de Junio de 2011

Aceptado: 23 de Enero de 2012

\section{Resumen}

La salinidad en los suelos es un problema ambiental global. La causa principal del problema es el manejo inadecuado que se realiza sobre los suelos, lo cual se conjuga principalmente con el contexto social rural. Se debe trabajar en la búsqueda de soluciones sostenibles viables que perciban la complejidad del problema en sus aspectos sociales, culturales, económicos, políticos y técnicos, desde la perspectiva de la Producción Más Limpia. Ésta es una apuesta que complementa investigación y desarrollo en función del mejoramiento de la calidad de vida de los habitantes de las regiones afectadas por salinidad, considerando la participación de diferentes actores sociales interesados. Propone complementar estudios analíticos y sintéticos de suelos como método de evaluación de las relaciones entre agua-suelo-planta-atmósfera; considera el saber tradicional y los métodos de la ciencia formal; e intenta complementar conservación (sostenibilidad ambiental) con producción (enfoque de producción más limpia).

Palabras claves: Complementariedad, Gestión de suelos, Producción más limpia, Potencial productivo del suelo, Salinidad del suelo.

\section{Abstract}

The salinity in soils is a global environmental problem. The main cause of the problem is the mismanagement that is performed on the soil, which is conjugated mainly with the rural social context. It is necessary to work in finding sustainable viable solutions perceiving the complexity of the problem in its social, cultural, economic, political and technical aspects, from the perspective of Cleaner Production. This is a bet that complements research and development in terms of improving the quality of life of the inhabitants of the regions affected by salinity, considering the participation of different concerned social actors. It proposes to complement analytical and synthetic soil's studies as a method for measuring the relations between water-soil-plant-atmosphere; considers traditional knowledge and methods of formal science and seeks to complement conservation (environmental sustainability) with production (cleaner production approach).

Keywords: Complementarity, Soil management, Cleaner production, Soil production potential, Soil salinity. 


\section{INTRODUCCIÓN}

La búsqueda de un sistema sostenible de producción agrícola basado en los principios de la producción más limpia (P.M.L.) en suelos afectados por salinidad es el objeto de estudio de este artículo. Este trabajo pretende analizar las relaciones de la sociedad con el medio ambiente alrededor de la desertificación de suelos por salinización, en la zona del distrito de riego Roldanillo - La Unión - Toro (R.U.T.), en el centro-norte del departamento del Valle del Cauca, Colombia, como caso de estudio.

La salinización puede darse de forma natural: cuando se trata de suelos bajos y planos, que son inundados por ríos o arroyos; o en aguas subterráneas poco profundas que ascienden por capilaridad conteniendo sales disueltas; por sales disueltas en las aguas de escorrentía; en las zonas costeras, por efecto del mar, a partir del nivel freático salino y por la contribución del viento. Las causas humanas corresponden a un manejo inadecuado de los terrenos, por ejemplo, por sales usadas en el agua de riego; o debido a un descenso del nivel freático, llevando a cabo la intrusión de aguas salinas.

La FAO estima que el $25 \%$ de las aéreas que utilizan sistemas de riego en zonas áridas y semiáridas del planeta están afectadas por procesos de degradación (Amezketa 2005). Según (Vázquez 2004) en el mundo existen 76,6 millones de hectáreas con problemas de salinización inducidas por el hombre, de un total de 1474 millones de hectáreas cultivables, es decir el 5,2\%. En el valle geográfico del río Cauca se evidencia altos contenidos de sales, con unas 85000 ha con suelos salinos de unas 200.000 ha analizadas, ubicadas principalmente en la zona del Distrito de Riego R.U.T. y Palmaseca (Palmira) (CVC \& Univalle 2009).

La región del Distrito R.U.T., por su origen de formación lacustre evidencia altos contenidos de sales, situación que se ha venido agravando por efecto de las prácticas agrícolas, además de las condiciones propias de la región (zonas con alta evapotranspiración y baja precipitación), suelos con tendencias arcillosas, mal drenaje y prácticas inadecuadas en su manejo (MADVT 2004).

El problema, de manera general, consiste en las consecuencias del incremento de intensidad de la salinización: se reduce el área cultivable y se afecta la economía campesina de la región. Aunque los cultivadores saben que están afectando el medio ambiente al transformarlo y que esto impulsa procesos de degradación de suelos (efecto osmótico, toxicidad, pérdida de estructura) también les preocupa la baja rentabilidad de los cultivos por el uso necesario de costosos insumos para contrarrestar el efecto negativo de la salinidad. Por las características de la comunidad se puede afirmar que, aunque las ganancias sean bajas, se requiere hacer agricultura en el terreno aun cuando las condiciones salinas se mantengan e incluso se incrementen, pues ellos necesitan sobrevivir.

\section{CONTEXTO AGRÍCOLA}

\section{Caso de estudio: Distrito de Riego Roldanillo - La Unión - Toro, RUT.}

En el Valle del Cauca se inició la construcción del distrito de riego R.U.T. desde el año 1958 lo que ha permitido un amplio desarrollo de cultivos de frutales, principalmente. El distrito RUT comprende unas 10.200 ha con unos 998 usuarios correspondientes a unas 6.500 familias, lo que genera unos 10.000 empleos directos y unos 40.000 indirectos.

El RUT queda ubicado a una altura de $900 \mathrm{msnm}$, con temperatura promedio de $26^{\circ} \mathrm{C}$ y unos $1.800 \mathrm{~mm}$ de precipitación al año. Se destaca por la amplia variedad de cultivos en relación al resto del departamento; se tiene unas 2.000 ha en frutales, 3.500 ha en caña de azúcar, 2.500 ha en maíz, además de unas 1.600 ha en otros variados cultivos que incluyen hortalizas (MADVT 2004).

El distrito RUT posee una gran infraestructura que incluye $156 \mathrm{~km}$ en canales de distribución manejados a través de dos gigantescas estaciones de bombeo desde el río Cauca y unas 400 compuertas; tiene $144 \mathrm{~km}$ de canales de drenaje apoyados en tres estaciones de bombeo, $75 \mathrm{~km}$ de diques para control de inundaciones y $385 \mathrm{~km}$ de vías de acceso y transporte de carga, pero esto no soluciona el problema en la agricultura.

El $60 \%$ de los usuarios son agricultores de bajos ingresos y poseen menos de 2 ha correspondientes a un $9 \%$ del total del área del distrito; mientras el $5 \%$ de usuarios posee el $65 \%$ del área con predios con más de 40 ha, lo que es una evidente inequidad social. Así, existen unas 3.000 familias con problemas económicos para la producción agropecuaria a nivel de microempresa; entre estas se tiene los propietarios de la finca Las Gramas de 38 ha que fue adjudicada en el 2003 a cinco unidades agrícolas familiares. "Las Parcelas" como comúnmente se les llama presentan algunas ventajas como organización y optimismo para mejorar su calidad de vida; los pobladores tienen la expectativa de adelantar proyectos agropecuarios.

Las consecuencias que sufren los pobladores del Distrito 
R.U.T. a causa de la salinización del suelo son, principalmente, pérdida de su fertilidad, la disminución de la infiltración y la toxicidad de algunas sales para los cultivos; igualmente reduce el crecimiento de las plantas y limita su productividad, generando pérdidas económicas a los agricultores (MADVT 2004) .

Un abordaje analítico tradicional de suelos no permite considerar la complementariedad entre la visión analítica y la visión sintética de un sistema agrícola (Zúñiga \& Pohlan 2001).

Un modelo de postdesarrollo plantea que se requiere ampliamente de un sano interés de la sociedad y de sus gobernantes por apoyar el modelo alternativo (Carvajal 2009) y en ésta empresa los científicos ambientales podemos realizar un papel protagónico.

Trabajar en profundidad en el sistema: ser parte del sistema para hacer la caracterización de los métodos de gestión de suelos del territorio es la propuesta para lograrlo. No se trata solo de detectar problemas y diseñar soluciones, sino de involucrarse en el fenómeno, aprendiendo a vivir y convivir con él e ir desarrollando mejores condiciones. Este esfuerzo requiere que la relación sea tan profunda que le sea inherente el deseo de potenciarse sinérgicamente con las transformaciones. Aquí se parte del hecho que la comunidad acepte a los investigadores y los haga partícipes del proceso.

\section{MARCO CONCEPTUAL}

\section{Sobre la salinidad en los suelos}

La contaminación química del suelo consiste en una degradación que provoca la pérdida parcial o total de la productividad del suelo como consecuencia de la acumulación de sustancias tóxicas en unas concentraciones que superan el poder de amortiguación natural del suelo y que modifican negativamente sus propiedades.

Un suelo salino posee una elevada cantidad de sales solubles, este tipo de sales son las que están en la disolución del suelo. Las sales solubles principales son cloruro y sulfato de sodio, magnesio, carbonatos de sodio y calcio. La salinización se refleja en un incremento en la conductividad eléctrica de la solución del suelo que tiene efectos adversos sobre las propiedades físicas y químicas del suelo, dificultando el crecimiento y la productividad vegetal (Ortiz \& Dorado 2007).

Existen varias clasificaciones de suelos salinos, cada una de las cuales con ventajas e inconvenientes. Las tres más importantes son la rusa, la francesa y la americana. La clasificación más simple y más práctica, se basa en la clasificación propuesta por el US Salinity Laboratory Richards, 1954 y perfeccionada por Massoud (1971). Esta clasificación utiliza fundamentalmente dos parámetros para caracterizar los suelos: la conductividad eléctrica (CE) y el porcentaje de sodio intercambiable (PSI).

La CE indica los efectos de la salinidad sobre las plantas. El PSI es un índice de los efectos sobre las propiedades del suelo. Los suelos se clasifican en tres categorías: suelos salinos, suelos sódicos y suelos salino-sódicos.

\section{Suelos salinos}

Se definen como los que contienen en la zona radicular una cantidad de sales disueltas en la solución del suelo (elevada CE) suficientemente alta para restringir el desarrollo de los cultivos. La reacción de estos suelos va de neutra a ligeramente alcalina. El $\mathrm{pH}$ puede variar entre 7 y menos de 8,5 . El PSI se mantiene por debajo de 7 , por lo que la estructura no se ve afectada (Sanchez \& Curetti 2005).

En la solución del suelo el sodio $(\mathrm{Na})$ rara vez representa más de la mitad de los cationes disueltos y, por tanto, no es adsorbido de forma importante. Los aniones principales son el cloruro y sulfato. Pueden presentarse también pequeñas cantidades de bicarbonato, pero invariablemente los carbonatos solubles casi no se encuentran.

Los suelos salinos casi siempre se reconocen por la presencia de costras blancas en su superficie. Estos suelos casi siempre se encuentran floculados, debido a la presencia de un exceso de sales en la solución y al reducido PSI. En consecuencia, su permeabilidad es igual o mayor a la de suelos similares no salinos.

Para manejo y/o recuperación se recomienda aplicación de enmiendas, que suelen ser químicas, en conjunto con el lavado de sales que consiste en la aplicación abundante de agua.

\section{Suelos sódicos}

Se definen como los que contienen en la zona radicular suficiente sodio adsorbido por el complejo de cambio para desarrollar propiedades físicas y químicas desfavorables, restringiendo el normal crecimiento de las plantas. La reacción de estos suelos varía según el PSl y la presencia o ausencia de $\mathrm{CO}^{=}$ó $\mathrm{CO}_{3}=$. El pH va desde 8 hasta más de 9,5. El contenido en sales de estos suelos es generalmente bajo (CEe $<2 \mathrm{mmhos} / \mathrm{cm}$ ). 
La solución del suelo contiene en su mayor parte cloruros, sulfatos y bicarbonatos, aunque puede haber pequeñas cantidades de carbonatos. A pH muy elevado y en presencia de iones carbonato, el calcio y el magnesio precipitan, por lo que las soluciones de los suelos sódicos contienen pequeñas cantidades de calcio y magnesio, predominando el sodio (Sanchez \& Curetti 2005).

En estos suelos las arcillas se dispersan; son arrastradas por el agua de lavado y pueden acumularse a pocos centímetros de profundidad, formando una capa pesada, de estructura prismática o columnar, poco permeable y asfixiante. La capa superior presenta textura gruesa y quebradiza. En los de elevado PSI, la materia orgánica se dispersa y disuelve, depositándose en la superficie, a la que da un color oscuro característico, dando origen a la denominación "álcali negro". Un elevado PSI afecta a las propiedades del suelo y no directamente a los cultivos, aunque indirectamente éstos se vean perjudicados por el deterioro de ciertas propiedades como estructura, permeabilidad, etc.

En los suelos sódicos no puede circular fácilmente el agua ni el aire, entonces como recomendación convencional de manejo es recomendable convertirlo a salino sódico, que tiene mayor permeabilidad; luego aplicar enmiendas y lavados. Esta estrategia es muy difícil de lograr y tiene elevadísimos costos.

\section{Suelos salino-sódicos}

Son aquellos que en la zona radicular contienen una cantidad de sales solubles, (medidas por la CEe), y un PSI suficientes para restringir el crecimiento de las plantas. Como límite se adoptan: CEe $>2 \mathrm{mmhos} / \mathrm{cm}$ y PSI $>7$. La reacción de estos suelos varía con su grado de salinidad, y con la presencia de $\mathrm{CO}=$ ó $\mathrm{CO}_{3}=$.

Siempre que contengan un exceso de sales (alta CEe), su apariencia y propiedades son similares a las de los suelos salinos. En ese caso el pH raramente es mayor de 8,5 y las partículas permanecen floculadas. Si el exceso de sales solubles es lavado, las propiedades de estos suelos pueden cambiar notablemente, llegando a ser idénticas a las de los suelos sódicos.

A medida que la concentración de sales disminuye en la solución, parte del sodio intercambiable se hidroliza para formar hidróxido de sodio que, a su vez, con el $\mathrm{CO}_{2}$ presente en la atmósfera del suelo puede formar $\mathrm{CO}_{3} \mathrm{Na}_{2}$. En cualquier caso, el lavado de un suelo puede hacerlo mucho más alcalino ( $\mathrm{pH}$ mayor de 8,5); las partículas coloidales se dispersan de la misma manera que en los suelos sódicos (Sanchez \& Curetti 2005).

\section{Métodos utilizados para diagnosticar y recuperar suelos afectados por salinidad}

Para convivir con el problema de salinidad se puede optar por cultivar plantas resistentes; manejar un alto nivel de humedad, cercano a capacidad de campo; o aplicar enmiendas y/o lavados que regulen los efectos de las sales en la fisiología vegetal. El método más usado es añadir yeso sobre la superficie, con lo cual se forma sulfato sódico que es una sal casi neutra y lavable.

El diagnóstico más utilizado para medir salinidad son los análisis de laboratorio de las muestras de suelo donde se analiza la conductividad eléctrica (CE) la cual debe ser superior a $4 \mathrm{dS} \mathrm{m} \mathrm{m}^{-1}$ a $25^{\circ} \mathrm{C}$. Sin embargo este método es costoso en tiempo, esfuerzo y dinero, cuando se trata de grandes áreas. Igualmente se utiliza la relación de adsorción de sodio (RAS) (Sanchez \& Curetti 2005).

El criterio tradicional para diferenciar entre suelos sódicos ha sido el de un porcentaje de saturación de sodio intercambiable (PSI) igual o mayor que un $15 \%$ del valor de la capacidad de intercambio catiónico (CIC). También se está utilizando un Sensor Electromagnético para medir la salinidad del suelo in situ, el cual mide la conductividad eléctrica aparente del suelo ( $\mathrm{CEa}$ ), que está estrechamente relacionada con la salinidad real del suelo (CEe) (MADVT 2004).

Desde el punto de vista de la recuperación, siempre es mucho más sencillo mejorar suelos salinos que aquellos con características sódicas. Mientras que los primeros pueden recuperarse con lavados, los sódicos precisan a su vez una enmienda que provea calcio. De todas maneras, para recuperar tanto un suelo salino como uno sódico es necesario un sistema de drenaje que garantice la eliminación de las sales del perfil del suelo (Sanchez \& Curetti 2005).

[En la presente investigación se propone explorar con el método de Reserva Energética del Suelo como su indicador de calidad y el método de Estimulación Magnética como práctica de conservación y/o recuperación de suelos degradados por salinidad.]

\section{Complementariedad: Producción más limpia y desarrollo humano sostenible en suelos afectados por salinidad}

Existe en la actualidad una dificultad evidente para aproximarse a la concepción del desarrollo sostenible, dificultad que tiene origen en la visión de desarrollo modernizante o reduccionista con que se ha mirado la naturaleza desde la concepción científica. 
El desarrollo modernizante está basado en una cosmovisión mecanicista caracterizada por el reduccionismo, su relación mecánica y no orgánica entre el todo y las partes, una visión determinista de los procesos, y una fragmentación total entre el sujeto y el objeto (Roldán et al. 2004).

El desarrollo humano sostenible en su concepción más amplia parte de una visión sintética, donde el todo es más que la suma de las partes, no existe una fragmentación entre el sujeto y el objeto: en el caso del medio ambiente no se considera al ser humano separado de la naturaleza, sino que ambos forman un todo indivisible. cita

Esta concepción del desarrollo humano sostenible enfatiza la síntesis en lugar del enfoque esencialmente analítico de la concepción modernizante. Sin embargo es innegable la necesidad del enfoque analítico y hay que reconocer todos los resultados positivos que ha producido. A pesar de que ambas concepciones del desarrollo sostenible y del modernizante, tienen aspectos mutuamente excluyentes, no parece que la disyuntiva sea una $\mathrm{u}$ otra. Ambas parecen ser de alguna manera necesarias. En este mismo sentido, la producción más limpia es una estrategia integrada a los procesos, a los productos y a los servicios, en búsqueda de reducir costos, incentivar innovaciones ambientalmente aceptables y reducir los riesgos relevantes para los seres humanos y el medio ambiente.

Existe entonces una aparente confrontación entre estas dos concepciones del desarrollo, lo cual aparentemente sumerge al investigador en una dicotomía donde se contraponen objetivos diferentes y al parecer contrapuestos. La mecánica Cuántica nos puede dar luz sobre este aparente paradigma. Según Bohr, dos conceptos son complementarios si son mutuamente excluyentes pero ambos necesarios para agotar toda la información, por ejemplo, los conceptos de onda y partícula, son conceptos complementarios respecto al electrón. Algunas veces el electrón se comporta como onda y otras como partícula. Bajo esta óptica de la complementariedad podría pensarse que las concepciones del desarrollo modernizante y del desarrollo sostenible que subyace en el quehacer de un investigador, aunque son mutuamente excluyentes pueden coexistir.

Igualmente para hacer gestión de suelos afectados por salinidad se debe complementar el talento humano de distintas disciplinas y ejes culturales. Esto implica reconocer que los problemas reales no son patrimonio de una disciplina, sino que requieren ser estudiados con los aportes de diferentes cosmovisiones y más aun de la propia comunidad.

Se requiere realizar un análisis de los sistemas agrícolas utilizados en el Distrito R.U.T. en búsqueda de implementar métodos apropiados a las características de los suelos agrícolas de la zona. El trabajo se debe realizar en el marco de la producción más limpia y el desarrollo humano sostenible, utilizando un enfoque analítico y sintético en la gestión de los suelos que incluye fertilización diferencial, evaluación de suelos, utilización de biofertilizantes, experiencias culturales, etc. tendiente a obtener un sistema racional de producción agrícola de mayor beneficio con menor deterioro medioambiental.

\section{HERRAMIENTAS DE PRODUCCIÓN MÁS LIMPIA Y DESARROLLO HUMANO SOSTENIBLE APLICADAS A SUELOS AFECTADOS POR SALINIDAD}

\section{Evaluación de la Reserva Energética del Suelo (RES) o Potencial Productivo del Suelo (PPS)}

Diversos estudios se han realizado para establecer la dinámica energética de los suelos, y poder definir los factores diferenciadores de su potencial productivo del suelo (PPS) o Reserva Energética del Suelo (RES). Con esta herramienta se ha avanzado en la construcción de una propuesta de agricultura de precisión, algunos de estos estudios se describen cronológicamente a continuación:

El concepto de reserva energética del suelo (RES), inició con su relación frente a la degradación del suelo en 200 ha con cultivo de caña de azúcar, bajo tres manejos de suelo: agroecológico, mixto y convencional (Gómez \& Romero 2004). Los datos arrojaron diferencias significativas entre el manejo agroecológico y convencional para la mayoría de las variables analizadas. Así, la reserva energética del suelo es un indicador cuantitativo de la calidad del suelo y permite diferenciar el efecto de las labores agrícolas desde un enfoque energético y holístico

En el 2005 se utilizó el concepto de potencial productivo del suelo (PPS) o reserva energética del suelo como indicador de calidad en un cultivo de maíz (Zea mays L.) Dekal B DK 5005 en El Cerrito Valle del Cauca, encontrando una correlación espacial positiva del $67 \%$ del PPS con la producción del cultivo (Duarte 2006).

En búsqueda de validación se evaluó el indicador del potencial productivo del suelo (PPS) o reserva energética del suelo frente a los análisis químicos de suelos en cultivos de caña de azúcar (Zúñiga et al. 2007), lo cual arrojó una correlación espacial positiva del $74 \%$. Además frente a las variables agronómicas, se obtuvo correlaciones de $89 \%$ y $61 \%$ del índice PPS con diámetro y altura de tallo del cultivo de caña de azúcar, respectivamente. 
Se realizó una zonificación de las características productivas del suelo estableciendo así una metodología para planificar las prácticas de conservación sobre estos suelos utilizando el potencial productivo del suelo o reserva energética del suelo en un sistema de manejo ecológico de yuca en el norte del Cauca (Zúñiga et al. 2008). Además se propuso una estrategia de fertilización diferenciada en las áreas de estudio que permite un ahorro en utilización de insumos para Caldono de 17,7\%, Santander 29,8\%, Caloto 32,2\% y Piendamó 17,1\%.

Se comparó el potencial productivo del suelo (PPS) o reserva energética del suelo en suelos con otros indicadores de calidad de suelos como el Índice de Fertilidad (IF) y el Índice de Compactación (IC) aplicado a suelos volcánicos de ladera de tres fincas lecheras (Nogales, Refugio y Horizonte) en Bolívar, Valle del Cauca (Zúñiga et al. 2009). La correlación lineal múltiple entre parámetros indicó que IC e IF influyeron aproximadamente en un $70 \%$ sobre PPS.

Se desarrolló un modelo de Redes Neuronales para determinar el índice Potencial Productivo del Suelo (PPS) o Reserva Energética del Suelo (RES) para predecir la variabilidad de la producción del cultivo de yuca, en tres tipos de suelos del norte del departamento del Cauca, Colombia (Jiménez 2010). Se determinaron correlaciones espaciales entre los mapas generados por los modelos desarrollados y los mapas de producción real, del orden de 0,38 para el lote ubicado en Caldono, 0,58 para el lote ubicado en Caloto y 0,40 para el lote ubicado en Santander de Quilichao. Resultados aceptables para una primera aproximación del modelo.

El cálculo de la RES se logra con la implementación de un modelo de redes neuronales a partir de parámetros sintéticos y analíticos para diagnosticar el potencial productivo del suelo. Se espera probar la técnica de la Reserva Energética del Suelo como indicador de calidad de suelos salinizados de tal manera que ofrezca una mayor precisión en la distribución espacial de los niveles de sales para así establecer las correspondientes acciones tecnológicas de recuperación.

\section{Estimulación electromagnética con fines agrícolas y su relación con la gestión de suelos salinos}

En la agricultura se ha empleado el tratamiento magnético al agua de riego para propiciar a la planta una mejor asimilación de nutrientes. También se ha empleado esta técnica para proporcionar un mejor crecimiento y desarrollo de las plantas. La aplicación del electromagnetismo se ha convertido en un fenómeno complejo, ya que encierra una serie de condiciones experimentales que abarca diferentes formas de aplicación (Díaz 2001).

Al ser los sistemas biológicos sistemas no lineales, no se pueden esperar los mismos resultados para todas las especies con las mismas características del campo aplicado (Serguera 2001).

Yakolev et al (1976, 1985), Zoltareva (1986), Shumakov et al (1989), Lin y Yotvat (1990), Gemishev y Todorov (1991), Milián et al (1991), Daidinov et al (1995), Monedero et al (1995), y Duarte C. (1995) citados por (Díaz 2001); evidencian la influencia de los campos magnéticos sobre el agua aplicada en riego de diferentes cultivos. Díaz (2001) concluyó que la magnetización con imanes permanentes del agua influye en la evaporación y conductividad de ésta.

Al evaluar la respuesta de dos variedades de ajo al tratamiento magnético del agua de riego, Duarte (2001) obtuvo rendimientos para las dos variedades evaluadas del orden del $11 \%$ a los $16 \%$, mayores, en comparación con el testigo.

Mujari, citado por (Carbonell \& Martínez 2001), manifiesta que el crecimiento de las raíces primarias del maíz se acelera con campos magnéticos alternos de frecuencias bajas.

Toda materia cuando es purificada y a su nivel molecular básico posee propiedades características que tienen resonancia a estímulos externos electromagnéticos. La resonancia magnética ejerce un proceso de antioxidación similar al que ocurre en el ciclo del agua, en este caso la resonancia magnética resultante es buena para el cuerpo y la salud, y ejerce una influencia pasiva en las criaturas vivas (Higa 2002).

Al observar los efectos de distintos tipos de tratamiento magnético en semillas de trigo (Triticum aestivum L.) bajo diferentes condiciones geográficas, climáticas y experimentales, sometiendo las semillas a la acción de campos magnéticos estacionarios creados por imanes, se obtuvo que el tratamiento magnético produce una estimulación del desarrollo de la planta, independientemente de las condiciones de aplicación, reflejada en los indicadores de crecimiento, fisiológicos y de rendimiento evaluados (Socorro et al. 2002).

En una investigación sobre la aplicación de tratamientos físico-químicos (activación y magnetización) para el riego del tomate variedad Rilia (Duarte et al. 2004) se observaron incrementos en los rendimientos del orden del $26 \%$ para el tratamiento con agua activada y del $45 \%$ para el tratamiento con agua tratada magnéticamente, con relación al tratamiento testigo donde se utilizó agua corriente. 
Se estudió el efecto del tratamiento magnético en la presiembra de semillas incrementando significativamente $(p<0,05)$ el crecimiento de la raíz, el tallo y el área foliar de las plantas e influyeron positivamente en la tasa relativa de crecimiento de la planta entera, en la producción de materia seca y en la distribución de la biomasa vegetal, y redujeron el tiempo (7 días) de permanencia de las posturas en semilleros (De Souza et al. 1999).

Con estimulación con campos magnéticos variables se disminuye significativamente el periodo de maduración de la cachaza utilizando tecnología electromagnética para uso como fertilizante (Cuero \& Tulande 2004). Para potenciar contundentemente el proceso de descomposición de la cachaza fresca se recomienda emplear estimulación magnética con campo magnético de $4.00 \mathrm{mT}$, producido a una frecuencia de $25 \mathrm{~Hz}$, durante al menos 120 minutos, con aplicaciones de microorganismos.

El tiempo de estimulación y la intensidad de campo electromagnético sobre el agua afectan la germinación y fortaleza de las semillas, esta manifestación se presenta más claramente a medida que se aumenta la intensidad de campo (Ipaz \& López 2004).

El riego con agua tratada magnéticamente reduce la diferencia entre los gastos de los emisores del sistema de riego, disminuyendo la variabilidad entre ellos y aumenta el coeficiente de uniformidad del riego (Duarte et al. 2005).

Empleando un tratamiento con 0,06 T de inducción magnética y un control se evaluaron las variables longitud del tallo, raíz y área foliar evidenciando que el empleo del agua tratada magnéticamente permite un mejor crecimiento y desarrollo a las estacas de romero, obteniéndose posturas vigorosas y de mejor calidad (Fung et al. 2007).

El campo magnético aceleró la acción dinámica de los microorganismos benéficos para descomponer moléculas complejas del material orgánico de la cachaza a sustancias más simples, facilitando la absorción de elementos hacia la planta (Peña 2010). Se aceleró el proceso natural de descomposición orgánica en un $49,1 \%$ de la cachaza fresca al aproximarla al grado de mineralización de la cachaza madura, lo cual demuestra la efectividad de la técnica electromagnética.

De acuerdo a (MADVT 2004), se recuperó suelos degradados por salinidad en el Valle del Cauca, obteniendo que el tratamiento con estimulación magnética con biofertilizantes al aumenta la producción del cultivo del maíz en un $45 \%$ con respecto al testigo absoluto. Estas variaciones de las propiedades del agua dependen de la intensidad y el gradiente del campo magnético, la velocidad y el movimiento del agua, El tamaño y forma de los polos, la instalación, la presencia de partículas coloides en el agua y la concentración de iones (MADVT 2004).

Los efectos del tratamiento magnético en sistemas acuosos provocan variaciones en las propiedades de éstos, conllevan a cambios en la cinética de todos los procesos físico-químicos y por tanto, en los procesos tecnológicos y biológicos. Estas variaciones generalmente son pequeñas, sin embargo, las consecuencias en los ámbitos industrial y biológico son muy significativas con esperanza de manejo de los suelos salinizados:

a) Hidratación de los iones. La fijación de la molécula de agua por los iones, ejerce una influencia muy grande en las propiedades de las soluciones y en la cinética de muchos procesos físico-químicos. El grado de hidratación se comporta en dependencia de la naturaleza del ión.

b) Conductividad eléctrica. Generalmente disminuye entre el 8 y $10 \%$ después del tratamiento magnético. No obstante existen informes en que se relata su aumento.

c) Tensión superficial. En general se produce disminución. Los cambios de ésta tienen un carácter completo observándose el valor máximo con una intensidad entre 560$640 \mathrm{KA} / \mathrm{m}$.

d) Solubilidad. Regularmente se plantea un aumento de la solubilidad de las sales en el agua tratada magnéticamente. En varias investigaciones se ha demostrado un aumento significativo de la solubilidad del carbonato de calcio. Semejantes resultados se obtuvieron con respecto a la solubilidad del oxígeno; hay autores que señalan aumento de la actividad del oxígeno disuelto.

e) Cristalización. Este aspecto está bastante estudiado. Se plantean cambios en la velocidad de cristalización, en la morfología, tamaño, forma etc. Como sustancia patrón clásica para todo estos estudios se tomó el carbonato de calcio.

f) Coagulación. Numerosos resultados experimentales evidencian un aumento significativo en la coagulación en los sistemas coloidales.

g) Memoria magnética. Una de las peculiaridades del tratamiento magnético en los sistemas acuosos son los cambios reversibles que se producen cuando el agua atraviesa al campo magnético. Es decir, después de un tiempo determinado por las condiciones externas, el sistema recupera su estado original. El tiempo durante el cual 
mantiene el sistema las variaciones en las propiedades con respecto a las originales, se denomina memoria magnética.

Al tratar electromagnéticamente los biofertilizantes, disminuye la tensión superficial del líquido en el suelo y aumentar la solubilidad de las sales, coadyuvando a la liberación de sales en el suelo por infiltración (sistema de drenaje) y así recuperando los suelos degradados por salinidad (MADVT 2004).

Generalmente la acción nutricional de los biofertilizantes sobre la planta es muy lenta a comparación de los fertilizantes químicos. Por lo tanto los agricultores tienden más a la aplicación de fertilizantes químicos aumentando la problemática de degradación de los suelos. La propuesta de uso de la estimulación magnética sobre suelos salinos busca acelerar la acción nutricional de los biofertilizantes sobre la planta, lo cual estimula la actividad de los microorganismos benéficos para que intervengan mineralizando más rápidamente los componentes orgánicos, facilitando la absorción de elementos hacia la planta.

La zona seleccionada para el trabajo, la finca Las Gramas (Roldanillo, Valle del Cauca) tiene problemas de salinidad que limitan la productividad, por lo tanto, es un ejemplo de las condiciones limitantes de pequeños agricultores beneficiarios de programas de reforma agraria.

Se propone, bajo un enfoque de complejidad y no linealidad, identificar las zonas homogéneas de Reserva Energética del Suelo para correlacionarlas con los grados de afectación del suelo por salinidad. Con base en este factor energético se realiza un diseño experimental que involucra las tecnologías convencionales y las tecnologías de estimulación electromagnética. El trabajo se evalúa mediante las características agronómicas, contexto socioeconómico alrededor del cultivo y propiedades físicas, químicas, biológicas y holísticas del suelo.

Del proyecto se espera obtener la metodología para: elaborar Mapas de la Reserva Energética del Suelo (RES) en relación con los Grados de Salinidad; diseñar planes de recuperación de suelos degradados por salinidad de acuerdo con los mapas de RES y bajo el enfoque de la Producción Más Limpia; estimar los impactos económicos de los diferentes tratamientos, los impactos ambientales y su relación con la participación activa comunitaria (productores, empresarios, comunidad académica y científica) de socialización de decisiones y resultados de los procesos implementando parcelas demostrativas.

\section{CONCLUSIONES Y RECOMENDACIONES}

En el Distrito de Riego R.U.T. es inminente la necesidad de implementar un sistema de producción agrícola más limpio para sus suelos afectados por salinidad. Se requiere evaluar métodos de gestión de suelos afectados por salinidad en búsqueda de adaptar o diseñar el o los más apropiados según las características de la región.

De acuerdo al mapa de RES se busca diferenciar las características de calidad del suelo a lo largo y ancho de la suerte, esto implica que se debe implementar una estrategia sectorizada de recuperación de suelos degradados por salinidad.

La utilización de métodos físico-energéticos del suelo debe permitir no sólo reforzar su competitividad sino también la sostenibilidad del sistema de producción, por cuanto la aplicación de la técnica de la Reserva Energética del Suelo permite elaborar planes de conservación y/o recuperación de suelos degradados por salinidad, también nos ayuda a elaborar planes de fertilización diferencial que contribuyen a reducir costos por las menores dosis aplicadas de fertilizantes por hectárea, y que también genera un impacto ambiental positivo, por la menor contaminación sobre los recursos naturales, suelo, aguas subterráneas, biodiversidad y salud de los operarios de campo.

Igualmente prácticas e insumos amigables con el ambiente como son los biofertilizantes estimulados electromagnéticamente, permiten el desarrollo de cultivos con un menor impacto negativo en su entorno a la vez que favorecen las sostenibilidad del cultivo en el espacio y en el tiempo.

Para que la selección de dichos métodos logre trascender se debe utilizar sistemas complejos de evaluación, para ello se propone aplicar métodos de análisis de suelos basados en el enfoque energético y la aceptación cultural. Por lo tanto hallar el mejor modelo productivo amigable con el ambiente permitirá conservar y mejorar las características originales de los suelos, contribuyendo así a la sostenibilidad de los recursos naturales y por consiguiente de los ecosistemas de Producción Más Limpia.

\section{REFERENCIAS}

AMEZKETA, E. 2005. "Vigilancia de la Salinidad en Suelos de Regadío mediante el Sensor Electromagnético", Departamento de Agricultura, Ganadería y Alimentación del Gobierno de Navarra, España. 
Ambiente y Sostenibilidad 2012 (2): 59-68.

Revista del Doctorado Interinstitucional en Ciencias Ambientales

ISSN: 2339-3122

CARBONELL, M. V. \& MARTÍNEZ, E. 2001. Bioelectromagnetismo aplicado a la agricultura. España.

CARVAJAL. A. 2009. "Desarrollo y postdesarrollo: modelos y alternativas". Facultad de Humanidades, Escuela de Trabajo Social y Desarrollo Humano, Universidad del Valle. Cali, Colombia. 265 pp.

CUERO. R. \& TULANDE, R. 2004. "Evaluación de la estimulación magnética con campo magnético variable en la cachaza seca y en el período de compostaje de la cachaza verde de caña de azúcar (Saccharum officinarum L.) tratadas con microorganismos benéficos". Trabajo de grado para optar el título de Ingeniero Agrícola. Universidad del Valle.

CVC \& UNIVALLE. 2009. “Diseño y promoción de tecnologías y prácticas para la recuperación de áreas con suelos degradados por erosión y salinidad". Cali, Colombia. $167 \mathrm{pp}$.

DE SOUZA A. PORRAS, L. R. \& CASATA, R. 1999. “Efecto del tratamiento magnético de semillas de tomate (Lycopersicon esculentum Mill) sobre la germinación y el crecimiento de las plántulas". Agronomía de Producción Vegetal 14:(3) 437-444.

DÍAZ, J. 2001. "Estudio de factores que afectan a la evaporación del suelo", Tesis doctoral. Universidad Politécnica de Madrid, Escuela Técnica Superior de Ingenieros Agrónomos.

DUARTE C. GUEVARA, G. \& MÉNDEZ, M. 2004. “Uso del agua activada y con tratamiento magnético del tomate en condiciones de organopónico". Universidad Agraria de La Habana, Cuba. Revista Ciencias Técnicas Agropecuarias 13 (003): 73-76.

DUARTE, C. 2001. "Respuesta de dos variedades de ajo al tratamiento magnético del agua de riego". Universidad Agraria de La Habana, Cuba. Revista Ciencias Técnicas Agropecuarias 10 (003): 73-76.

DUARTE, C. RODRÍGUEZ, R. REY, R. L. GONZÁLEZ, L. \& GUEVARA, G. 2005. “Algunas consideraciones sobre el uso del agua tratada magnéticamente en especies ornamentales del Jardín Botánico Nacional". Universidad Agraria de La Habana, Cuba. Revista Ciencias Técnicas Agropecuarias 14 número (001): 37-41.

DUARTE, L. 2006. "Desarrollo de una metodología de evaluación del potencial productivo del suelo como indicador de calidad en un cultivo de maíz (Zea mays L.)
DekalB DK- 5005 en Cerrito Valle del Cauca". Tesis de pregrado Ingeniería Agrícola. Universidad del Valle.

FUNG, Y. ISAAC, E. \& FERRER, A. 2007. “Estudio en el crecimiento de plantas de Rosmarinus officinalis regadas con agua tratada magnéticamente". XVII Con. Ven. Bot. Centro Nacional de Electromagnetismo Aplicado (CNEA), Santiago de Cuba, Cuba.

GÓMEZ, P. ROMERO, G. 2004. “Evaluación de la reserva energética de suelos en tres sistemas agronómicos del cultivo de caña de azúcar Cerrito - Valle. Tesis de pregrado Ingeniería Agrícola Universidad del Valle.

HIGA, T. 2002. “Una revolución para salvar la Tierra: una forma de resolver los problemas de nuestro mundo a través de los microorganismos efectivos (EM)". Gràfiques Manlleu, España. 332 pp.

IPAZ, C. \& LÓPEZ, M. 2004. “Evaluación de la germinación y la primera fase de crecimiento del cultivo de sorgo hibrido 744 (Sorghum vulgare), bajo el efecto del uso de un sistema dinámico de estimulación electromagnética para el agua y microorganismos en solución. Trabajo de grado en Ingeniería Agrícola, Universidad del Valle.

JIMÉNEZ, C. 2010. “Modelación del potencial productivo del suelo de cultivos de yuca (Manihot esculenta Crantz) en zonas de ladera del norte del Cauca, a partir de propiedades del suelo y rendimientos. Trabajo de grado en Ingeniería Agrícola. Universidad del Valle.

MADVT, 2004. Plan de Acción Nacional de lucha contra la desertificación y la sequía en Colombia. 33 pp.

MASSOUD, F. I. 1971. A note on the need for accepted definitions and methods of characterization of salt-affected soils. Reunión de la subcomisión de suelos salinos, Sevilla IRDA.

ORTIZ, S. \& DORADO, V. 2007. “Técnicas de Recuperación de Suelos Contaminados", Universidad de Alcalá, Informe de Vigilancia Tecnológica, Mid.

PEÑA, J. 2010. "Estimulación magnética de microorganismos benéficos aplicados a la cachaza para mejorar su uso como fertilizante orgánico". Universidad del Valle, COLCIENCIAS. Cali.

ROLDÁN, J. BEN-DOV, \& GUERRERO, G. 2004. "La complementariedad: una filosofía para el Siglo XXI". Universidad del Valle, Cali - Colombia. 
SANCHEZ, E. \& CURETTI, M. 2005. “Los suelos salinos y sódicos, características y diferenciación, modos de recuperación y recomendaciones".

SERGUERA, N. M. 2001. "Riegos con agua tratada magnéticamente". Centro Internacional de Electromagnetismo Aplicado (CENEA), Santiago de Cuba, Cuba.

SOCORRO, A. CARBONELL, M. V. MARTÍNEZ, E. PÉREZ, S. \& AMAYA, J. M. 2002. "Tratamiento magnético de semillas de trigo (Triticum aestivum L.) como técnica de estimulación del crecimiento". Revista Alimentaria 337: 167170.

VÁZQUEZ, R. B. 2004. “Influencia de la salinidad sobre el suelo". Escuela Universitaria de Ingeniería, Valladolid.

ZÚÑIGA, O. \& POHLAN, J. 2001. “Agricultura orgánica en Colombia un enfoque analítico y sintético". Universidad del Valle, Cali - Colombia. 401 pp.

ZÚÑIGA, O. CUERO, R. ERAZO, E. TORRES, R. \& DÁVILA, G. 2008. “Determinación del potencial productivo del suelo en un cultivo de yuca a partir de la medición de la conductividad térmica y resistividad eléctrica del suelo en el norte del Cauca". Suelos Ecuatoriales 38(1): 9-15.

ZÚÑIGA, O. CUERO, R. OSORIO, J. J. ZAPATA, J. CAICEDO, J. \& ROA, A. 2007. "Metodología para determinar el potencial productivo del suelo a partir de la medición de la conductividad térmica y su relación con los análisis químicos en un cultivo de caña de azúcar en el Valle del Cauca". Suelos Ecuatoriales 37(1): 10-14.

ZÚÑIGA, O. MADERO, E. ROJAS, A. \& RAMÍREZ, L. 2009. "Índice de potencial productivo del suelo aplicado a tres fincas". Acta Agronómica 58(2): 85-90. 